\title{
Evaluation of Distribution Features of Bone Metastases by Bone Scintigraphy in Prostate Cancer
}

\author{
Ashraf Anas Zytoon ${ }^{1}$, Eman Abdel Razek Tawfek ${ }^{2}$, Adel Mohamed Elwakil ${ }^{1}$, Gilan Ibrahim Aladle ${ }^{1}$ \\ ${ }^{1}$ Radiology Department, Faculty of Medicine, Menoufia University, Menoufia, Egypt \\ ${ }^{2}$ Nuclear Medicine \& Clinical Oncology Department, Faculty of Medicine, Menoufia University, Menoufia, Egypt
}

Email address:

ashradio@gmail.com (A. A. Zytoon), agamaleldeen@yahoo.com (E. A. R. Tawfek), Adel.elwakeel221@yahoo.com (A. M. Elwakil), gilanaladle2010@yahoo.com (G. I. Aladle)

\section{To cite this article:}

Ashraf Anas Zytoon, Eman Abdel Razek Tawfek, Adel Mohamed Elwakil, Gilan Ibrahim Aladle. Evaluation of Distribution Features of Bone Metastases by Bone Scintigraphy in Prostate Cancer. International Journal of Medical Imaging. Vol. 3, No. 6, 2015, pp. $137-142$.

doi: $10.11648 /$ j.jimi.20150306.15

\begin{abstract}
Prostate cancer (PCa) represents the most common non-cutaneous malignancy for men, where an estimated 241,740 new cases will be diagnosed in 2013 in the United States [1]. Whereas most of those new diagnoses will be clinically localized, up to $17 \%$ of patients may experience metastatic disease, in which the risk of cancer- specific mortality is increased [2]. In this context, it is well established that, beyond regional lymph nodes, the skeleton represents the most common metastatic site [3]. The study aims to evaluate the pattern of distribution of bone metastases in prostate cancer patients by 99mTc- methylene diphosphonate (99mTc-MDP) bone scintigraphy. It was formed in 150 patients with pathologically proven prostate cancer and presented for bone scans with $99 \mathrm{mTc}-\mathrm{methylene}$ diphosphonate (99mTc-MDP) for detection and evaluation of bone metastases. It was noted that patients with bone metastases had significantly higher frequency of bone pain when compared with patients without and noted that spine was the most commonly affected site (60.0\%) followed by ribs $(52.0 \%)$ and femur $(30.0 \%)$. Spread of bony metastases among the bony skeleton has distribution manner not only to the right or to the left. Metastatic bony lesions of prostate cancer are located mainly in spine (58.0\%) followed by ribs (52.0\%) and femur $(30.0 \%)$. Patients with extensive bone metastasis had significantly higher frequency of skull, spine, scapula, humerous, sternum, ribs, iliac bone, ischium, and femur metastases.
\end{abstract}

Keywords: Prostate Cancer, Bone Scan, Bone Metastases, MDP

\section{Introduction}

Prostate cancer is the most common male cancer diagnosed in Western population. Autopsy studies have shown that with increasing age the majority of men will develop microscopic foci of cancer in populations at both high and low risk. However, only a small percentage of men will develop invasive form of prostate cancer. The cancer cells may metastasize from the prostate to other parts of the body, particularly the bones and lymph nodes [4].

The presence of prostate cancer may be indicated by symptoms, physical examination, prostate-specific antigen (PSA), or biopsy.Prostate- specific antigen testing increases cancer detection but does not decrease mortality [5].

Prostate cancer is the second most frequently diagnosed cancer of men after lung cancer and the fifth most common cancer worldwide [6]. It constitutes $10.6 \%$ of cancers of men in sub- Saharan Africa [7]. In the USA the tumor appears to be more common in African-American men than in Caucasian men [8].

Prostate cancer frequently metastasizes to bone but metastases can also be found in other body organs and tissues [9]. They contribute significantly to the morbidity associated with advanced disease [10]. The bone metastases of prostate cancer are usually radiologically osteoblastic. Presentation with diffuse osteolytic bone metastases is rare and only a few case reports exist in the medical literature with this type of metastatic lesion[11,12].

Bone scintigraphy is a sensitive and efficient method for the detection of bone metastases of malignant tumors, although it is considered to be a nonspecific method. Bone scintigraphy has value in determining the stage [13]. Selecting therapeutic schedule, evaluating prognosis [14] and performing follow-up [15] for tumor patients. 


\section{Aim of the Work}

The aim of the study is to evaluate the pattern of distribution of bone metastases in prostate cancer patients by 99mTc- methylene diphosphonate (99mTc-MDP) bone scintigraphy.

\section{Patient and Methods}

This study was performed between August 2014 andAugust 2015. This research was done after faculty committee approval (available upon request). Furthermore, this study is a retrospective study. It included 150 patients with cancer prostate. They had a mean age of $61.2 \pm 10.2$ years (40 - 86 years). They were referred to Clinical Oncology and Nuclear Medicine Departments, Menofyia University for bone scan.

\subsection{Methods}

Whole-body bone scans, anterior and posterior views obtained with a dual detector gamma camera were studied from 150 patients who had undergone scintigraphy, with Tc99m methylene diphosphonate(600 MBq Tc- 99m MDP) because of suspected bone metastatic disease.

All patients were subjected to full history taking, Clinical examination, assessment of PSA, MRI pelvis. CT chest, Biopsy and Gleason score and Staging of all patients.

\subsection{Diagnostic Criteria of Bone Metastasis}

The results of bone scans were interpreted as bone metastasis if radiotracer activity of local bone lesion was greater or less than that of contralateral or adjacent normal bone tissues.

The results of bone scans were considered negative under the following circumstances:

a) No abnormal increased radiotracer uptake was detected.

b) Radiotracer uptake was characterized by medical\& benign disease (e.g. arthritis, fracture, and trauma)

\subsection{Classification of Bone Metastasis}

The lesions in the body were divided into three categories depending on their location: left, center, and right sides. Lesions in the center were located in the vertebrae and chest bone, those on the left side were located on left parts of the body from the middle line, except the vertebrae and chest bone, and those on the right side were located on the right parts of the body from the middle line, except the vertebrae and the chest bone. According to the total number of metastatic bony lesions, patients were divided into three groups: fewer bone metastases, with the total number being one to three; moderate bone metastases, with the total number being four to 10; and extensive bone metastases, with the total number being more than 10 [16].

\section{Results}

Data management: Statistical analysis

- Data obtained from the present study were computed using SPSS versions 17 under the platform of Microsoft Windows XP, Professional Edition.

- Continuous data were expressed in the form of mean \pm $\mathrm{SD}$ while categorical data were expressed in the form of count and percent.

- Comparison of continuous data were performed utilizing student $t$ test, while categorical data were done using Chi-square test.

- $\mathrm{P}$ value less than 0.05 was considered statistically significant.

In the present study, the studied patients had a mean age of $61.2 \pm 10.2$ years. Among the studied patients, 67 patients (44.7\%) had irritative symptoms while 25 patients $(16.7 \%)$ had obstructive symptoms and 95 patients $(63.3 \%)$ had bone pain (Figure 1).

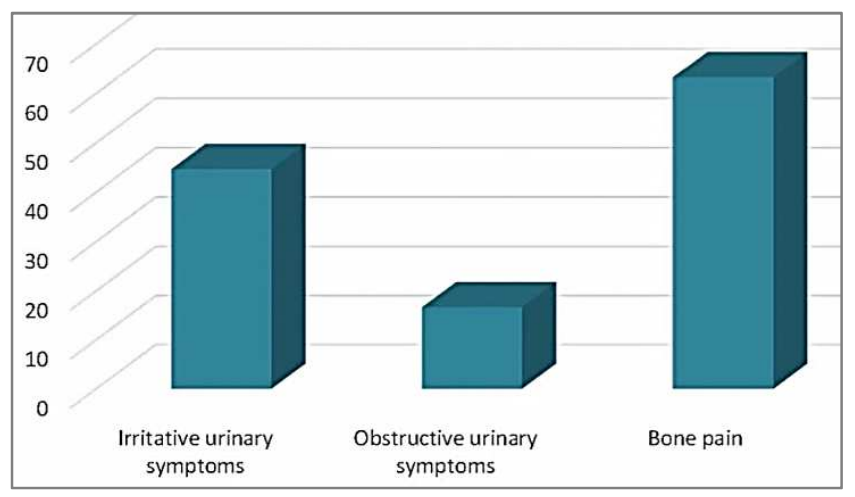

Figure 1. Reported presentations in the studied patients.

In the present study, it was noted that spine was the most commonly affected site $(60.0 \%)$ followed by ribs $(52.0 \%)$ and femur (30.0\%) (Table 1).

Table 1. Distribution of bone metastasis in the studied patients $(n=100)$.

\begin{tabular}{lll}
\hline & No & \% \\
\hline Skull & 13 & 13.0 \\
Spine & 60 & 60.0 \\
Scapula & 11 & 11.0 \\
Humerus & 20 & 20.0 \\
Clavicle & 11 & 11.0 \\
Sternum & 6 & 6.0 \\
Ribs & 52 & 52.0 \\
Iliac Bone & 20 & 20.0 \\
Sacrum & 9 & 9.0 \\
Acetabulum & 10 & 10.0 \\
Ischium & 8 & 8.0 \\
Pubis & 7 & 7.0 \\
Femur & 30 & 30.0 \\
Tibia & 3 & 3.0 \\
\hline
\end{tabular}

As regards the grade of bone metastases in the studied patients, we found that the studied patients 61 patients with 
few bone metastases, 29 patients with moderate metastases and 10 patients with extensive bone metastases (Figure2).

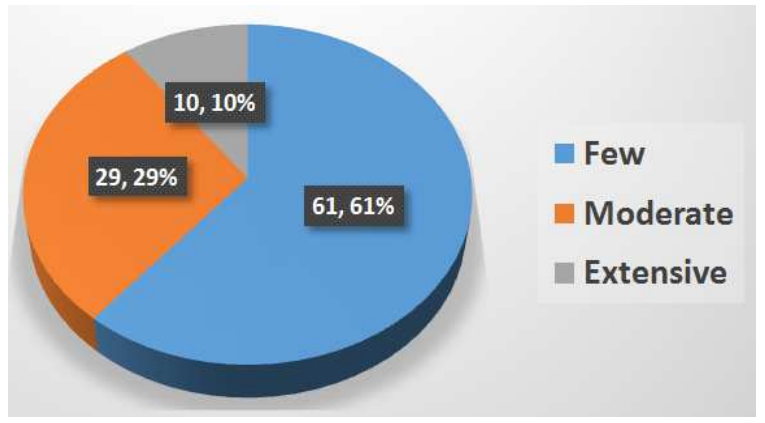

Figure 2. Grade of bone metastases in the studied patients.In the current study, we found that patients with bone metastases had significantly higher frequency of bone pain when compared with patients without (Table 2).

Table 2. Comparison between patients with and without bone metastases regarding clinical presentations, regardless presence or absence of visceral metastases.

\begin{tabular}{llllll}
\hline & $\begin{array}{l}\text { Patients with } \\
\text { bone metastases }\end{array}$ & $\begin{array}{l}\text { Patients without } \\
\text { bone metastases }\end{array}$ & \multicolumn{2}{l}{ Chi-square test } \\
\cline { 5 - 6 } & $\mathbf{n}=\mathbf{5 0}$ & $\mathbf{X 2}$ & $\mathbf{p}$ \\
\hline $\begin{array}{l}\text { Irritative } \\
\text { symptoms }\end{array}$ & 47 & 20 & 0.66 & 0.42 \\
$\begin{array}{l}\text { Obstructive } \\
\text { symptoms }\end{array}$ & 20 & 5 & 2.4 & 0.12 \\
Bone pain & 75 & 20 & 17.6 & $0.0001^{*}$ \\
\hline
\end{tabular}

In the current study, patients with extensive bone metastasis had significantly higher frequency of skull, spine, scapula, humerous, sternum, ribs, iliac bone, ischium, and femur metastases when compared with patients with moderate metastases (Table 3).

Table 3. Comparison between patients with few and moderate metastases regarding metastases location.

\begin{tabular}{llllll}
\hline & Few & Moderate & Extensive & \multicolumn{2}{c}{ Chi-square test } \\
\cline { 5 - 7 } & $\mathbf{N = 6 1}$ & $\mathbf{n = 2 9}$ & $\mathbf{n = 1 0}$ & $\mathbf{X 2}$ & $\mathbf{p}$ \\
\hline Skull & 5 & 4 & 4 & 7.7 & $0.021^{*}$ \\
Spine & 30 & 20 & 10 & 10.6 & $0.005^{*}$ \\
Scapula & 3 & 5 & 3 & 7.1 & $0.028^{*}$ \\
Humerus & 8 & 6 & 6 & 9.0 & $0.003^{*}$ \\
Clavicle & 1 & 5 & 5 & 22.2 & $0.0001^{*}$ \\
Sternum & 2 & 2 & 2 & 4.3 & 0.12 \\
Ribs & 26 & 17 & 9 & 8.4 & $0.015^{*}$ \\
Iliac Bone & 4 & 10 & 6 & 20.7 & $0.0001^{*}$ \\
Sacrum & 5 & 2 & 2 & 1.7 & 0.43 \\
Acetabulum & 6 & 2 & 2 & 1.42 & 0.49 \\
Ischium & 2 & 2 & 4 & 15.8 & $0.0001^{*}$ \\
Pubis & 1 & 2 & 4 & 19.3 & $0.0001^{*}$ \\
Femur & 10 & 13 & 7 & 16.0 & $0.0001^{*}$ \\
Tibia & 1 & 1 & 1 & 2.0 & 0.35 \\
\hline
\end{tabular}

Case No.1:

Bone scans of a 58-year-old patient with prostate cancer shows

Increase radiotracer activity over:

- Right Clavicle

- Spine: C7, D1 and D12
- Left 6th, 7th and 10th ribs

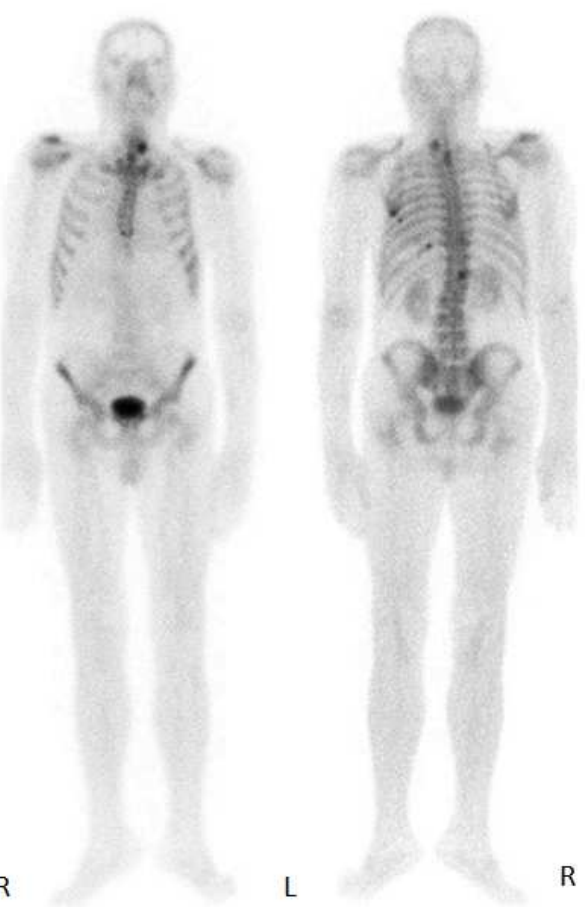

Case No.2:

Bone scans of a 67-year-old patient with prostate cancer shows

Increase radiotracer activity over:

- Both Shoulders more dominant left

- Spine: D 3, 11 \& 12 and L 1, 2, 3, 4 \& 5

- Left 7th, 9th ribs, right 11 th rib

- Both sacroiliac joints

- Right and left femur head, both greater trochanters and lower end of left femur

- Upper part of left tibia
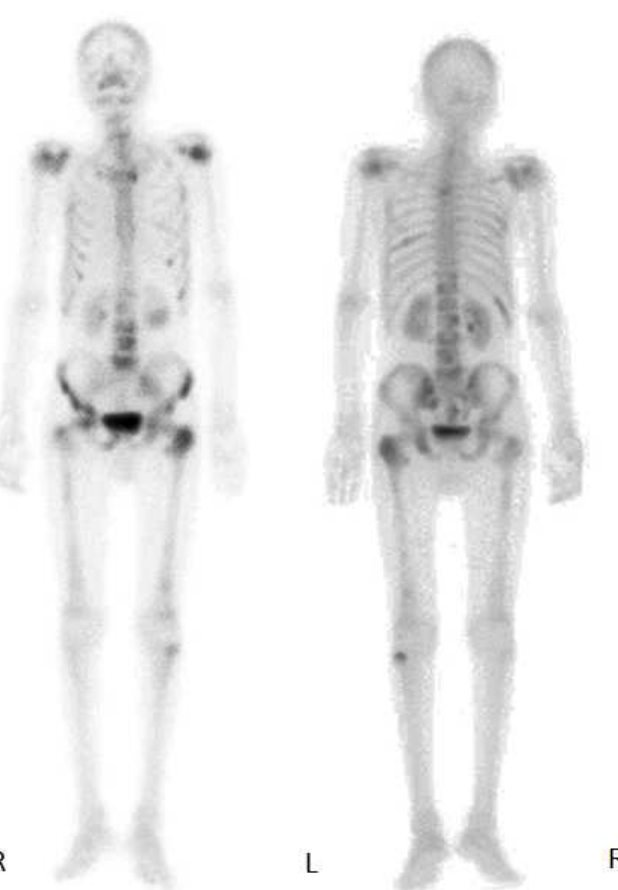

$\mathrm{L}$

$\mathrm{R}$ 
Case No.3:

Bone scans of a 64-year-old patient with prostate cancer shows

Increase radiotracer activity over:

- Medial end of right clavicle

- Right pubic bone

- Both humeri

- Both sacroiliac joints and Right iliac bone

- Spine D 12 and L 2, 3, 4 \& 5

- Right 8th, 9th\& 11th and Left 10th ribs

- Sacrum

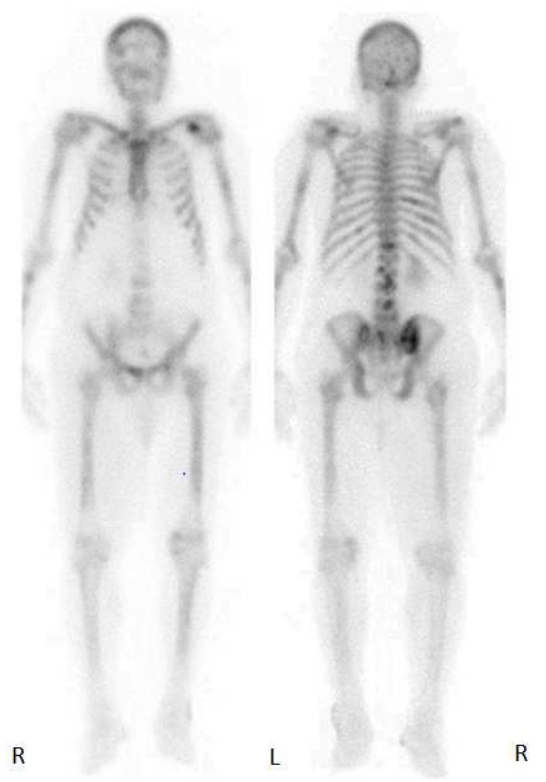

Case No.4:

Bone scans of a 70-year-old patient with prostate cancer shows

Increase radiotracer activity over:

- Spine: D7 \& D8

- Left 8th rib posteriorly

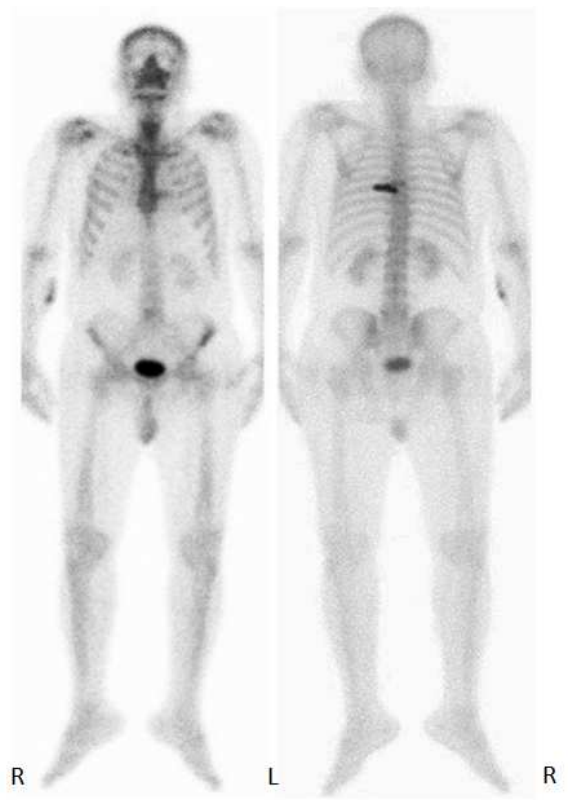

Case No.5:

Bone scans of a 54-year-old patient with prostate cancer shows

Increase radiotracer activity over:

- Medial end of right $3 \mathrm{rd} \& 7$ th ribs posteriorly

- Focal uptake at L1

- Head and neck of right femur

- Left femoral head

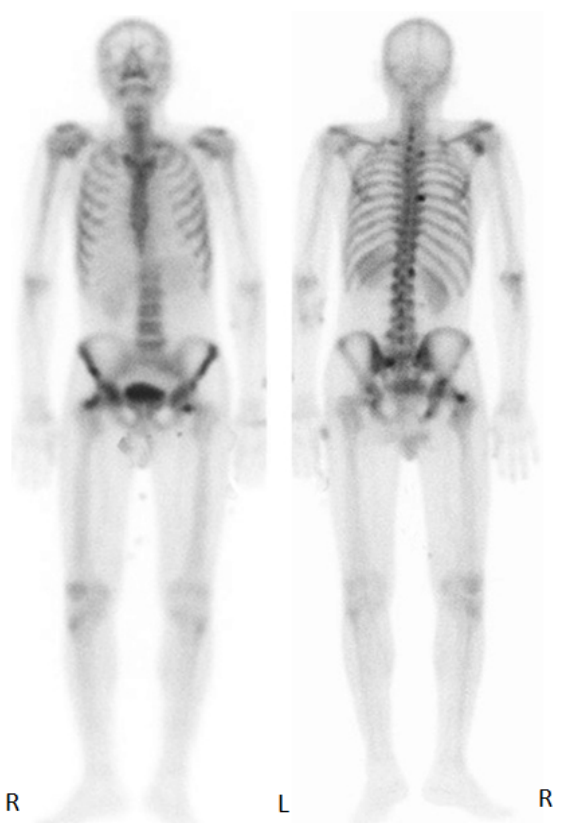

Case No.6:

Bone scans of a 67-year-old patient with prostate cancer shows

Increase radiotracer activity over:

- Right iliac and acetabulum

- Both pubic bones

- Lower part of both femurs

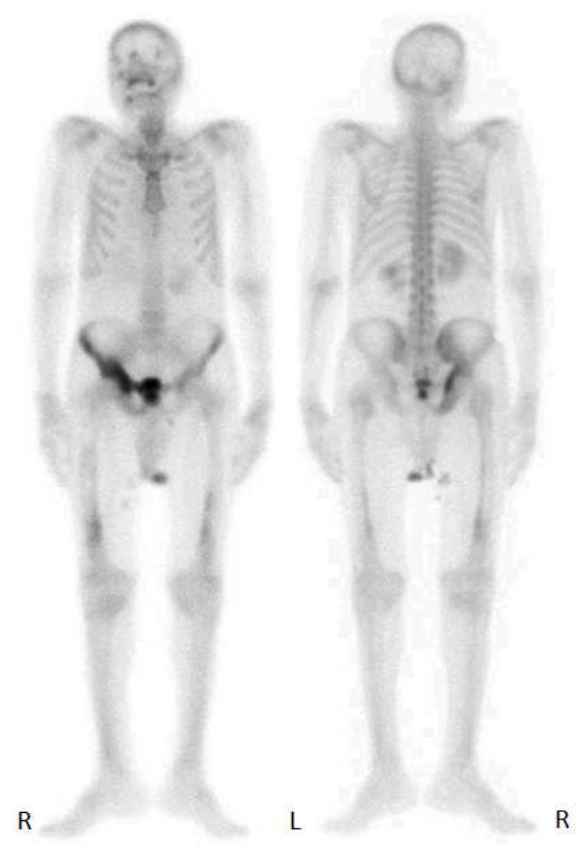




\section{Discussion}

Skeletal scintigraphy has long been a cornerstone of disease assessment in prostate cancer. $99 \mathrm{mTc}$-methylene diphosphonate (99mTc- MDP) bone scans are widely used and provide an indirect measure of tumor activity because they detect tracer deposition by osteoblasts along bone mineralization fronts. Prostate cancer bone metastases can be imaged this way because they are associated with elevated activity by both osteoblasts and osteoclasts. Further, 99mTcMDP bone scans are an established component of disease assessment in prostate cancer clinical trials [17].

The indication for bone scintigraphy can be a routine examination at the time of the diagnosis of cancer, but bone pain is also a common reason to perform an examination [18].

Many scholars have studied radionuclide bone imaging of prostate cancer, but their analysis in spreading regularity of bone metastasis is still not specific enough [19].

In the present study, the studied patients had a mean age of $61.2 \pm 10.2$ years. Among the studied patients, 67 patients $(44.7 \%)$ had irritative symptoms while 25 patients $(16.7 \%)$ had obstructive symptoms and 95 patients $(63.3 \%)$ had bone pain. These data are differs from that found by the study of [20] who aimed to report the pattern of prostate cancer presentation in Alexandria University. In this study, the most common clinical presentation was irritative symptoms. This may be explained by the high frequency of patients with bone metastases in the present study.

Regarding PSA levels, we found that among the studied patients, $84(56.0 \%)$ had a PSA of less than 10 and 66 patients $(44.0 \%)$ had PSA $\geq 10$. In respect to Gleason score, $40(26.7 \%)$ had a Gleason score of less than 6,33 patients (22.0\%) had a score from 6-7 and 77 patients $(51.3 \%)$ had a score more than 7 . This is in agreement with the study of [21].

In the current study, 100 patients $(66.7 \%)$ out of screened 150 had metastatic bone disease. This figure is lower than that found by the study of [22]. In this study, the authors systematically described the most common sites of metastases in a contemporary cohort of PC patients. Overall, 74,826 patients with metastatic $\mathrm{PCa}$ were identified. The most common metastatic sites were bone (84\%).In the present study, it was noted that spine was the most commonly affected site $(60.0 \%)$ followed by ribs $(52.0 \%)$ and femur $(30.0 \%)$. This is in accordance with the study of [16]. In their study, the authors investigated the distribution characteristics of bone metastases in breast and prostate carcinomas. Bone scintigraphies were performed in 504 cancer patients. They found that spine was the most common bone metastases site in patients with prostate cancer.

This is also in agreement with the study of [23] who tried to investigate the distribution of bone metastases in common cancers using bone scintigraphy. 160 consecutive patients with malignancy (prostate cancer: 32, breast cancer: 107, lung cancer:8, and gastrointestinal cancers: 13) underwent bone scan. In patients with cancer prostate, the most frequently involved area was the spine, followed by ribs and pelvic bones.

As regards the grade of bone metastases in the studied patients, we found that the studied patients 61 patients with few bone metastases, 29 patients with moderate metastases and 10 patients with extensive bone metastases. This varied from the findings of [16]. In their study, the authors explored the distribution features of metastatic bony lesions in prostate cancer. Bone scans with $99 \mathrm{mTc}-$ methylene diphosphonate were performed in 144 patients with pathologically proven prostate cancer, and distribution regularity of metastatic bony lesions was analyzed retrospectively. Among the 144 patients, 31 presented fewer bone metastases with a total of 58 lesions, 26 presented moderate bone metastases with a total of 161 lesions, and 45 presented extensive bone metastases with a total of 1781 lesions.

In the current study, we found that that patients with bone metastases had significantly higher frequency of bone pain when compared with patients without in agreement with the study of [18] who examined the relation between pain and bone metastases in a group of patients with prostate or breast cancer that had been referred for bone scintigraphy. Wholebody bone scans, anterior and posterior views obtained with a dual detector gamma camera were studied from 101 consecutive patients who had undergone scintigraphy (600 $\mathrm{MBq}$ Tc- 99m MDP) because of suspected bone metastatic disease. In patients with prostate cancer, they found metastases in $47 \%(18 / 38)$ of the patients with pain, but only in $12 \%(2 / 17)$ of the patients without pain.

In the current study, patients with extensive bone metastasis had significantly higher frequency of skull, spine, scapula, humerous, sternum, ribs, iliac bone, ischium, and femur metastases when compared with patients with moderate metastases. This agrees with the study of [16] .

\section{Conclusions}

- Prostate cancer frequently develops into bone metastasis. This study indicates that spine was the most commonly affected site by bone metastases followed by ribs and femur and shows that patients with bone metastases had significantly higher frequency of bone pain when compared with patients without.

- Our results show no significant differences between the studied groups regarding PSA levels and patients' age.

- patients with extensive bone metastasis had significantly higher frequency of skull, spine, scapula, humerous, sternum, ribs, iliac bone, ischium, pubis and femur metastases when compared with patients with moderate and few metastases. Spine was the most commonly affected site by bone metastases followed by ribs and femur.

Patients with bone metastases had significantly higher frequency of bone pain when compared with patients without.

\section{References}

[1] Siegel R, Naishadham D, Jemal A. Cancer statistics, 2013. CA: Cancer J Clin 2013; 63(1): 11-30. 
[2] Scosyrev E, Messing EM, Mohile S, et al. Prostate cancer in the elderly: Frequency of advanced disease at presentation and disease-specific mortality. Cancer 2012; 118(12): 3062-3070.

[3] Hess KR, Varadhachary GR, Taylor SH, et al. Metastatic patterns in adenocarcinoma. Cancer 2006; 106(7): 1624-1633.

[4] Russel P.J, P.Jackson,E.A. Kingsley. Prostate cancer methods and protocols. Methods in Molecular Medicine.2003; 81: 1-19.

[5] Djulbegovic M, Beyth RJ, Neuberger MM, et al. Screening for prostate cancer: systematic review and meta-analysis of randomised controlled trials. BMJ.2010; 341: c4543.

[6] Ferlay J, Shin HR, Bray F, et al. Cancer incidence and mortality worldwide. IARC Cancer Base No. 10.Int Agency Res Canc 2010.

[7] Freddy S, Max P, Zvavahera C, et al. Cancers. In Disease and Mortality in Sub-Saharan Africa. World Bank; 2006. ${ }^{\perp}$

[8] Danley KL, Richardson JL, Bernstein L, et al. Prostate cancer: trends in mortality and stage-specific incidence rates by racial/ethnic group in Los Angeles County, California (United States).Cancer Causes Control 1995; 6(6): 492-498.

[9] Vinjamoori AH, Jagannathan JP, Shinagare AB, et al. A typical metastases from prostate cancer: 10-year experience at a single institution. Am J Roentgenol 2012; 199(2): 367-372.

[10] Saad F, Clarke N, Colombel M. Natural history and treatment of bone complications in prostate cancer.Eur Urol 2006; 49(3): 429-440.

[11] Rajendiran G, Green L, Chhabra G. A rare presentation of prostate cancer with diffuse osteolytic metastases and PSA of 7242 ng/ml. Int J Case Rep Image 2011; 2(9): 16-20. ${ }^{\perp}$

[12] Migita T, Maeda K, Ogata N. A case of prostate cancer associated with osteolytic bone metastases.Hinyokika Kiyo 1999; 45(5): 371-374.

[13] Lin K, Szabo Z, Chin BB, Civelek AC. The value of a baseline bone scan in patients with newly diagnosed prostate cancer. Clin Nucl Med. 1999; 24: 579-582.
[14] Conti G, La Torre G, Cicalese V, et al. Prostate cancer metastases to bone: Observational study for the evaluation of clinical presentation, course and treatment patterns. Presentation of the metauro protocol and of patient baseline features. Arch Ital Urol Androl. 2008; 80: 59-64.

[15] Maffioli L, Florimonte L, Pagani L, et al. Current role of bone scan with phosphonates in the follow-up of breast cancer. Eur J Nucl Med Mol Imaging. 2004; 31(Suppl 1): S143-S148.

[16] Wang CY, Wu GY, Shen MJ, et al. Comparison of distribution characteristics of metastatic bone lesions between breast and prostate carcinomas. Oncol Lett. 2013 Jan; 5(1): 391-397.

[17] Scher B, Seitz M, Albinger W, et al. Value of PET and $\mathrm{PET} / \mathrm{CT}$ in the diagnostics of prostate and penile cancer. Recent Results Cancer Res. 2008; 170: 159-79.

[18] Levren G, Sadik M, Gjertsson P, et al. Relation between pain and skeletal metastasis in patients with prostate or breast cancer. Clin Physiol Funct Imaging. 2011 May; 31(3): 193-5.

[19] Helyar V, Mohan HK, Barwick T, et al: The added value of multislice SPECT/CT in patients with equivocal bony metastasis from carcinoma of the prostate. Eur J Nucl Med Mol Imaging 2010; 37: 706-713.

[20] Elabbady A, Eid A, Fahmy A, et al. Pattern of prostate cancer presentation among the Egyptian population: A study in a single tertiary care center. Cent European J Urol. 2014; 67(4): 351-6.

[21] Pal RP, Thiruudaian T, Khan MA. When is a bone scan study appropriate in asymptomatic men diagnosed with prostate cancer? Asian J Androl. 2008 Nov; 10(6): 890-5.

[22] Gandaglia G, Abdollah F, Schiffmann J, et al. Distribution of metastatic sites in patients with prostate cancer: A populationbased analysis. Prostate. 2014 Feb; 74(2): 210-6.

[23] Kakhki VR, Anvari K, Sadeghi R, et al. Pattern and distribution of bone metastases in common malignant tumors. Nucl Med Rev Cent East Eur. 2013; 16(2): 66-9. 